\title{
THE ISOMORPHISM THEOREM OF KLEINIAN GROUPS
}

\author{
Katsuhiko MatsuzAKI
}

Department of Mathematics, Ochanomizu University

\begin{abstract}
A sufficient condition on a geometrically finite Kleinian group $G$ is shown, under which any type-preserving isomorphism from $G$ onto another geometrically finite one is induced by an automorphism of the Riemann sphere.
\end{abstract}

The Fenchel-Nielsen isomorphism theorem asserts that a type-preserving isomorphism $\varphi: \Gamma \rightarrow \Gamma^{\prime}$ between cofinite volume Fuchsian groups $\Gamma$ and $\Gamma^{\prime}$ is induced by an automorphism $f$ of the unit disk $\Delta$, that is, there is $f$ such that $\varphi(\gamma)=f \circ \gamma \circ f^{-1}$ for every $\gamma \in \Gamma$. Roughly speaking, this means that an algebraic isomorphism between such Fuchsian groups is geometric. In this note, we extend this result to Kleinian groups and investigate a sufficient condition for an algebraic isomorphism to be geometric. Along this line, there is a result due to Marden and Maskit [7]. Their theorem works under certain assumptions on both the Kleinian group $G$ and the isomorphism $\varphi$. Our theorem assumes nothing about $\varphi$ but that it is type-preserving, and provides a sharp sufficient condition for $G$ under which any type-preserving isomorphism $\varphi$ is geometric.

A fundamental result is the following Marden isomorphism theorem [6].

Proposition. Let $G$ be a geometrically finite torsion-free Kleinian group, and let $\varphi: G \rightarrow G^{\prime}$ be a type-preserving isomorphism onto another Kleinian group. Suppose there is a homeomorphism $f: \Omega(G) \rightarrow \Omega\left(G^{\prime}\right)$ of the region of discontinuity where $f \circ g=\varphi(g) \circ f$ for all $g \in G$. Then $f$ extends to $\hat{\mathbb{C}}$ as an automorphism conjugating $g$ into $\varphi(g)$.

We may regard this proposition as a translation of the following topological result due to Waldhausen (cf. [2] Chap.13) into the Kleinian group theory: for compact orientable irreducible 3-manifolds $M$ and $M^{\prime}$ with incompressible boundary components, an isomorphism $\varphi: \pi_{1}(M) \rightarrow \pi_{1}\left(M^{\prime}\right)$ is geometric (i.e. there exists a homeomorphism $f: M \rightarrow M^{\prime}$ which induces $\varphi$ ) whenever it preserves the peripheral structure (i.e. for each component $S$ of $\partial M$, there is a component $S^{\prime}$ of $\partial M^{\prime}$ such that $\varphi$ maps $\pi_{1}(S)$ to a conjugate of $\pi_{1}\left(S^{\prime}\right)$ in $\left.\pi_{1}\left(M^{\prime}\right)\right)$. Hence the problem is reduced to a problem when the peripheral structure is preserved. Johannson [4] proved that if $M$ is acylindrical, then the peripheral structure is preserved. Our result may be regarded as a translation of Johannson's into the Kleinian group 
theory. However, without assuming his theorem, we exhibit in this note a simple proof relying on the intersection property of the limit sets of Kleinian groups, which was studied by Susskind [11].

Now, letting $\Omega(G)$ be the region of discontinuity of a Kleinian group $G, \Lambda(G)$ the limit set, and $\operatorname{Stab}_{G}(\Delta)$ the component subgroup for a component $\Delta$ of $\Omega(G)$, we state our result:

Theorem. Let $G$ and $G^{\prime}$ be geometrically finite Kleinian groups possibly with torsion. We assume that $G$ satisfies the following three conditions:

(0) each component $\Delta$ of $\Omega(G)$ is simply connected;

(1) G has no APT;

(2) for any distinct components $\Delta_{1}$ and $\Delta_{2}$ of $\Omega(G), \operatorname{Stab}_{G}\left(\Delta_{1}\right) \cap \operatorname{Stab}_{G}\left(\Delta_{2}\right)$ contains no loxodromic elements.

Then, for any type-preserving isomorphism $\varphi: G \rightarrow G^{\prime}$, there is an automorphism $f$ of $\hat{\mathbb{C}}$ such that $\varphi(g)=f \circ g \circ f^{-1}$.

Remark. The combination of the assumptions (0) and (1) is equivalent to the following condition:

$\left(1^{\prime}\right) G$ is a web group, i.e. every component of $\Omega(G)$ is a Jordan domain.

We can rewrite Theorem as a statement for hyperbolic manifolds. Let $\mathbb{H}^{3}$ be the hyperbolic 3-space, and $N_{G}$ a complete hyperbolic 3-manifold $\mathbb{H}^{3} / G$ divided by a finitely generated torsion-free Kleinian group $G$. When the convex core of $N_{G}$ has finite hyperbolic volume, we say that $G$ and $N_{G}$ are geometrically finite. We may regard $\Omega(G) / G$ as boundary at infinity of the hyperbolic manifold $N_{G}$. Consider the topological manifold $M_{G}=\left(\mathbb{H}^{3} \cup \Omega(G)\right) / G$ with boundary. Then the assumption (0) is equivalent to the condition that every boundary component $S$ of $M_{G}$ is incompressible, that is, the homomorphism $\pi_{1}(S) \rightarrow \pi_{1}\left(M_{G}\right)$ induced by the inclusion $S \hookrightarrow M_{G}$ is injective. In virtue of the loop theorem, we may say that $M_{G}$ has no essential disks when this condition is satisfied. The assumption (1) is equivalent to the following condition: if a loop in $\partial M_{G}$ is freely homotopic to a loop round a cusp in $M_{G}$, then the homotopy can be performed in $\partial M_{G}$. The assumption (2) is equivalent to the condition that $M_{G}$ is acylindrical: if two loops in $\partial M_{G}$ are freely homotopic in $M_{G}$, then the homotopy can be performed in $\partial M_{G}$ or they are freely homotopic to a loop round a cusp. In virtue of the annulus theorem, we may say that $M_{G}$ has no essential punctured-disks when the former condition is satisfied and no essential annuli when the latter is.

Theorem'. Let $N_{G}$ and $N_{G^{\prime}}$ be geometrically finite hyperbolic 3-manifolds. We assume that $N_{G}$ has neither essential disks, essential punctured-disks nor essential annuli. Then for any isomorphism $\varphi: \pi_{1}\left(N_{G}\right) \rightarrow \pi_{1}\left(N_{G^{\prime}}\right)$ which preserves the cusps, there is a (quasi-isometric) homeomorphism $f: N_{G} \rightarrow N_{G^{\prime}}$ which induces $\varphi$.

Remark. If we drop any of three assumptions in the above Theorem, we can find a counterexample to the statements. In this sense, our theorem is sharp. 
Proof of Theorem. Suppose that $G$ is torsion-free. If $G$ or $G^{\prime}$ is of the first kind, namely, $N_{G}$ or $N_{G^{\prime}}$ is of finite volume, then the Mostow rigidity theorem implies ours (cf. [6]). Hence we may further assume that $G$ and $G^{\prime}$ are of the second kind. Let $\Delta$ be any component of $\Omega(G)$ and $H$ the component subgroup $\operatorname{Stab}_{G}(\Delta)$. By the assumptions (0) and (1), we know $H$ is quasifuchsian. We shall prove that $H^{\prime}=\varphi(H)$ is also a component subgroup of $G^{\prime}$. This means that the peripheral structure is preserved by $\varphi$. Then our claim follows from the Marden isomorphism theorem (See [5] p.218 for a detailed argument to apply Marden's theorem).

First, we see that $H^{\prime}$ is also quasifuchsian. Indeed, the image $H^{\prime}$ under the typepreserving isomorphism is either quasifuchsian or totally degenerate ([9] Theorem 6 ), but it cannot be totally degenerate because $G^{\prime}$ is geometrically finite and of the second kind (cf. [10] p.134).

Next, we will show that $g^{\prime}\left(\Lambda\left(H^{\prime}\right)\right) \cap \Lambda\left(H^{\prime}\right)$ is empty or consists of one parabolic fixed point for any $g^{\prime} \in G^{\prime}-H^{\prime}$. When $\Lambda\left(H^{\prime}\right)$ satisfies this (and $h^{\prime}\left(\Lambda\left(H^{\prime}\right)\right)=\Lambda\left(H^{\prime}\right)$ for any $\left.h^{\prime} \in H^{\prime}\right)$, we say that $\Lambda\left(H^{\prime}\right)$ is precisely $H^{\prime}$-invariant except for a parabolic fixed point. We investigate the intersection of the limit sets of two subgroups in a Kleinian group. By the following lemma, which is a corollary to Susskind's result, we know that $\Lambda\left(H^{\prime}\right)$ satisfies the above property.

Lemma. Under the assumptions of Theorem, let $H_{1}$ and $H_{2}$ be distinct component subgroups of $G$. Then $\Lambda\left(\varphi\left(H_{1}\right)\right) \cap \Lambda\left(\varphi\left(H_{2}\right)\right)$ is empty or consists of one parabolic fixed point.

Proof. Since $\varphi\left(H_{1}\right)$ and $\varphi\left(H_{2}\right)$ are geometrically finite subgroups of a Kleinian group $G^{\prime}$, we know from Theorem 3 in [11] that

$$
\Lambda\left(\varphi\left(H_{1}\right)\right) \cap \Lambda\left(\varphi\left(H_{2}\right)\right)=\Lambda\left(\varphi\left(H_{1}\right) \cap \varphi\left(H_{2}\right)\right) \cup P^{\prime},
$$

where $P^{\prime}$ is a set of points fixed by a parabolic abelian group of rank 2 generated by an element of $\varphi\left(H_{1}\right)$ and another element of $\varphi\left(H_{2}\right)$. However $P^{\prime}$ is empty in our case. In fact, a parabolic element of $H_{1}$ and another of $H_{2}$ cannot generate an abelian group of rank 2 because $H_{1}$ and $H_{2}$ are component subgroups. Accordingly, one of $\varphi\left(H_{1}\right)$ and another of $\varphi\left(H_{2}\right)$ cannot, which implies that $P^{\prime}=\emptyset$. As a consequence, we have

$$
\Lambda\left(\varphi\left(H_{1}\right)\right) \cap \Lambda\left(\varphi\left(H_{2}\right)\right)=\Lambda\left(\varphi\left(H_{1}\right) \cap \varphi\left(H_{2}\right)\right)=\Lambda\left(\varphi\left(H_{1} \cap H_{2}\right)\right) .
$$

Here, $H_{1} \cap H_{2}$ is an elementary group without a loxodromic element by the assumption (2), and so is $\varphi\left(H_{1} \cap H_{2}\right)$. Therefore $\Lambda\left(\varphi\left(H_{1} \cap H_{2}\right)\right)$ consists of one parabolic fixed point at most, which proves the statement of the lemma.

Proof continued. We will see that $H^{\prime}$ is embedded, namely, there is a properly embedded incompressible surface $S^{\prime}$ in $N_{G^{\prime}}$ whose fundamental group is $H^{\prime}$ under the identification $\pi_{1}\left(N_{G^{\prime}}\right) \cong G^{\prime}$. Since $\Lambda\left(H^{\prime}\right)$ is precisely $H^{\prime}$-invariant except for a parabolic fixed point, we can construct an $H^{\prime}$-invariant and $G^{\prime}$-equivariant contractible 
surface in $\mathbb{H}^{3}$ with the boundary $\Lambda\left(H^{\prime}\right)$ (cf. [10] VII.B.16). Then its projection to $N_{G^{\prime}}$ yields the desired $S^{\prime}$.

As the final step of the torsion-free case, we will show that $H^{\prime}=\varphi(H)$ is a component subgroup of $G^{\prime}$. If not, the properly embedded incompressible surface $S^{\prime}$ induces a non-trivial amalgamated or HNN free product decomposition of $G^{\prime}$. It is

$$
G^{\prime}=\Gamma_{1}^{\prime} \underset{H^{\prime}}{*} \Gamma_{2}^{\prime} \quad \text { or } \quad G^{\prime}=\Gamma_{H^{\prime}}^{*}
$$

according as $M_{G^{\prime}}-S^{\prime}$ is disconnected or connected. Then, operating $\varphi^{-1}$, we have a non-trivial decomposition

$$
G=\Gamma_{1} \underset{H}{*} \Gamma_{2} \quad \text { or } \quad G=\Gamma_{H}^{*} \underset{H}{*} .
$$

Let $\left(M_{G}\right)_{0}$ be the pared manifold $M_{G}-\{$ cusp neighborhoods $\}$. It is a compact topological manifold with boundary whose interior is homeomorphic to $N_{G}$. Using the above free product decomposition of $G \cong \pi_{1}\left(\left(M_{G}\right)_{0}\right)$, we have a properly embedded incompressible surface $S$ in $\left(M_{G}\right)_{0}$ such that $\pi_{1}(S)$ corresponds to a subgroup of $H$ and $S$ induces a non-trivial decomposition of $G$ (cf. [3] p.35). Further, since $\varphi$ is type-preserving, all parabolic elements of $G$ are contained in conjugates of the factors of this decomposition. Hence, by moving $S$ by a homotopy if necessary, we may assume that $\partial S$ is in the non-cuspidal boundary $\partial_{n}\left(M_{G}\right)_{0}=\partial\left(M_{G}\right)_{0} \cap \partial M_{G}$. If $\partial S$ is not empty, every component of $\partial S$ must be in the surface $\Delta / H$ because an annulus $(A, \partial A)$ in $\left(\left(M_{G}\right)_{0}, \partial_{n}\left(M_{G}\right)_{0}\right)$ is not essential due to the assumptions (1) and (2). If $S$ were a disk, then $\partial_{n}\left(M_{G}\right)_{0}$ would be compressible. This contradicts the assumption (0), and thus $S$ is not a disk. Since every non-trivial loop in $S$ is freely homotopic to a loop in $\Delta / H$, we can see that $S$ divides $\left(M_{G}\right)_{0}$ into two parts, one of which is homeomorphic to $S \times[0,1]$. This contradicts the fact that $S$ induces a non-trivial amalgamated free product decomposition of $G$. Thus the proof of the torsion-free case completes.

In case $G$ contains elliptic elements, we take a torsion-free subgroup $\Gamma$ of $G$ with finite index by the Selberg lemma. Since $\Lambda(\Gamma)=\Lambda(G)$, $\Gamma$ also satisfies the assumptions (0), (1) and (2). We restrict the isomorphism $\varphi$ to $\Gamma$. Then $\left.\varphi\right|_{\Gamma}: \Gamma \rightarrow \Gamma^{\prime}$ is geometric by the result in the torsion-free case; there is an automorphism $f$ of $\hat{\mathbb{C}}$ which induces $\left.\varphi\right|_{\Gamma}$. In particular, $f$ determines the correspondence between the components $\Delta$ of $\Omega(\Gamma)=\Omega(G)$ and $\Delta^{\prime}$ of $\Omega\left(\Gamma^{\prime}\right)=\Omega\left(G^{\prime}\right)$. In each component $\Delta$, we modify $\left.f\right|_{\Delta}$ so that it may compatible with $H=\operatorname{Stab}_{G}(\Delta)$. This is possible because the type-preserving isomorphism $\left.\varphi\right|_{H}$ is geometric by the original Fenchel-Nielsen isomorphism theorem for Fuchsian groups. Thus we can construct a homeomorphism $\tilde{f}: \Omega(G) \rightarrow \Omega\left(G^{\prime}\right)$ which induces $\varphi: G \rightarrow G^{\prime}$. Consider $f^{-1} \circ \tilde{f}$. It is defined on $\Omega(\Gamma)$ and induces the identity isomorphism $\Gamma \rightarrow \Gamma$. Then by the Maskit identity theorem [8], $f^{-1} \circ \tilde{f}$ is extendable to an automorphism of $\hat{\mathbb{C}}$, and so is $\tilde{f}$. This completes the proof of the general case.

Remark. In [5], Keen, Maskit and Series have shown that for a geometrically finite web group $G$ such that every component of $\Omega(G)$ is a round disk, the peripheral 
structure is preserved under any type-preserving isomorphism onto another geometrically finite Kleinian group $G^{\prime}$. It is evident that such a Kleinian group $G$ satisfies our assumptions (0), (1) and (2). The authors use two facts to prove their result: a lemma due to Otal and a theorem by Floyd [1]. The former lemma characterizes whether a quasifuchsian subgroup of $G^{\prime}$ is peripheral or not in terms of the topology of the limit set. The latter theorem asserts that $\Lambda(G)$ and $\Lambda\left(G^{\prime}\right)$ are homeomorphic if $G$ and $G^{\prime}$ are isomorphic under a type-preserving map. We can see that their arguments extend to another proof of our theorem.

\section{REFERENCES}

1. W. Floyd, Group completions and limit sets of Kleinian groups, Invent. Math. 57 (1980), 205-218.

2. J. Hempel, 3-manifolds, Ann. Math. Studies 76, Princeton Univ. Press, 1976.

3. W. Jaco, Lectures on three-manifold topology, CBMS Regional Conference Ser. 43, AMS, 1977.

4. K. Johannson, Homotopy equivalences and 3-manifolds with boundaries, Lecture Notes 761, Springer, 1979.

5. L. Keen, B. Maskit and C. Series, Geometric finiteness and uniqueness for Kleinian groups with circle packing limit sets, J. reine angew. Math. 436 (1993), 209-219.

6. A. Marden, The geometry of finitely generated Kleinian groups, Ann. of Math. 99 (1974), 383-462.

7. A. Marden and B. Maskit, On the isomorphism theorem for Kleinian groups, Invent. Math. 51 (1979), 9-14.

8. B. Maskit, Self-maps on Kleinian groups, Amer. J. Math. 93 (1971), 840-856.

9. B. Maskit, On the classification of Kleinian groups: I-Koebe groups, Acta Math. 135 (1976), 249-270.

10. B. Maskit, Kleinian groups, Springer, 1988.

11. P. Susskind, Kleinian groups with intersecting limit sets, J. Analyse Math. 52 (1989), 26-38.

Otsuka 2-1-1, Bunkyo-Ku, TOKYo 152, JAPAN

E-mail address: matsuzak@math.ocha.ac.jp 